\title{
The Contact State Monitoring for Seal End Faces Based on Acoustic Emission Detection
}

\author{
Xiaohui Li, ${ }^{1}$ Pan Fu, ${ }^{1}$ Kan Chen, ${ }^{2}$ Zhibin Lin, ${ }^{1}$ and Erqing Zhang ${ }^{1}$ \\ ${ }^{1}$ School of Mechanical Engineering, Southwest Jiaotong University, Chengdu 610031, China \\ ${ }^{2}$ Sichuan Sunny Seal Co. Ltd, Chengdu 610041, China
}

Correspondence should be addressed to Pan Fu; pfu@home.swjtu.edu.cn

Received 19 July 2015; Revised 20 October 2015; Accepted 21 October 2015

Academic Editor: Peng Chen

Copyright (c) 2016 Xiaohui Li et al. This is an open access article distributed under the Creative Commons Attribution License, which permits unrestricted use, distribution, and reproduction in any medium, provided the original work is properly cited.

\begin{abstract}
Monitoring the contact state of seal end faces would help the early warning of the seal failure. In the acoustic emission (AE) detection for mechanical seal, the main difficulty is to reduce the background noise and to classify the dispersed features. To solve these problems and achieve higher detection rates, a new approach based on genetic particle filter with autoregression (AR-GPF) and hypersphere support vector machine (HSSVM) is presented. First, AR model is used to build the dynamic state space (DSS) of the AE signal, and GPF is used for signal filtering. Then, multiple features are extracted, and a classification model based on HSSVM is constructed for state recognition. In this approach, AR-GPF is an excellent time-domain method for noise reduction, and HSSVM has advantage on those dispersed features. Finally experimental data shows that the proposed method can effectively detect the contact state of the seal end faces and has higher accuracy rates than some other existing methods.
\end{abstract}

\section{Introduction}

As a kind of noncontact seal, hydrodynamic seal works by a thin fluid film between the pair of end faces for sealing and lubricating [1]. But when the seal is just started up, the film cannot be formed immediately, and the two end faces still keep contact with each other. This contact will cause continuous scratching and wearing and produce strong force and heat to reduce the working life of the seal. From the above, monitoring the contact state of seal end faces would help the early warning of the seal failure.

For monitoring the face contact, the fluid film has been measured via approaches of capacitance and resistance [2,3], and eddy current method has also proved to be useful [4]. However, since the seal pair should be modified for sensors installation, these methods can only be taken in laboratory.

Ultrasonic detection is another classical method for mechanical seal, and the film thickness can be estimated based on the ultrasonic attenuation $[5,6]$. However, considering the sensors' cost, this method is also unsuited for wide application.

Considering nondestruction and low cost, acoustic emission $(\mathrm{AE})$ detection has a better application prospect, and this method has been well applied on many machines [7-9]. But for seal, there are very few successful cases. The most major problem of this method is difficult to isolate the seal signal from background noise $[10,11]$, and this makes the existing methods cannot work well in practice [12-14].

The purpose of this paper is to develop a reliable approach to monitor the seal face contact by $\mathrm{AE}$ detection. For the problem of the difficulties in signal denoising and features classification, a new approach based on genetic particle filter with autoregression (AR-GPF) and hypersphere support vector machine (HSSVM) is presented. In this processing, AR model is used to build the dynamic state space (DSS) of the AE signal, and GPF is used for signal filtering. Then following feature extraction, a classification model based on HSSVM is constructed. Data shows that the proposed approach can effectively detect the contact state of the seal end faces and has higher accuracy rates than some other existing methods.

\section{Related Work}

For mechanical seal, the AE occurs when the equipment is distorted, being rubbed or under collision. In order to extract 


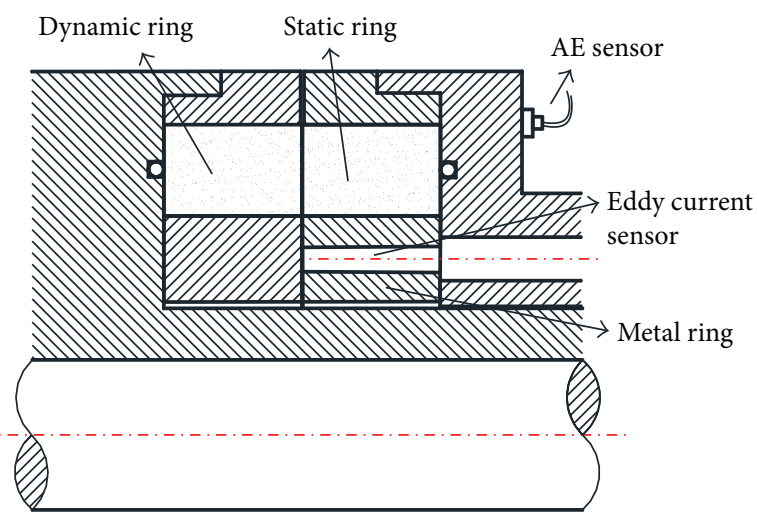

FIGURE 1: Sensors installation.

useful information, some new studies and methods have been attempted in recent years.

Fan et al. have processed the AE signal by multiple signal classification with higher order statistics (HOS-MUSIC) [12] and extracted the information about seal end faces under noisy condition. But in his case, the noise circumstance is artificial, and the paper's focus is not on the state monitoring of the seal end faces. Gao et al. have studied the AE characteristics of dry gas seal during the seal startup and used RMS of the signal to describe the friction strength of the seal end faces [15]. However, no denoising method has been presented in their paper, and the unique feature is not reliable enough to detect the seal face contact. Zhang et al. have described the seal face contact in the view of tribology and used Laplace wavelet and its coherent coefficient [13] for state monitoring. Then for improving the detection rates, another method based on wavelet packet and radial basis function (RBF) artificial neural network (ANN) [14] has been presented. But because of the unstable spectrum structure of the seal signals and the dispersed distribution of the $\mathrm{AE}$ features, both the methods will be ineffective when random contact occurs. Particle filter is a time-domain method for signal denoising, and this method has been applied to measure the film thickness [16]. However, since the particle diversity reduces during the resampling procedure, this method cannot reach the best performance.

Compared with the above methods, AR-GPF is a timedomain method with higher denoising performance, and the model based on HSSVM has more advantage in dealing with the features under dispersed distribution.

\section{Experiment and Signal Observation}

3.1. Experiment. We take hydrodynamic mechanical seal as research object and water as sealing medium. The sensors installation is given by Figure 1 .

In Figure 1, an eddy current sensor is used to measure the film thickness. In order not to damage the seal end faces, a metal ring with a through hole is inlaid in the static ring for sensor installation. The type of the eddy current sensor is $\mathrm{KD} 2360$, and its resolution is $0.1 \mu \mathrm{m}$.

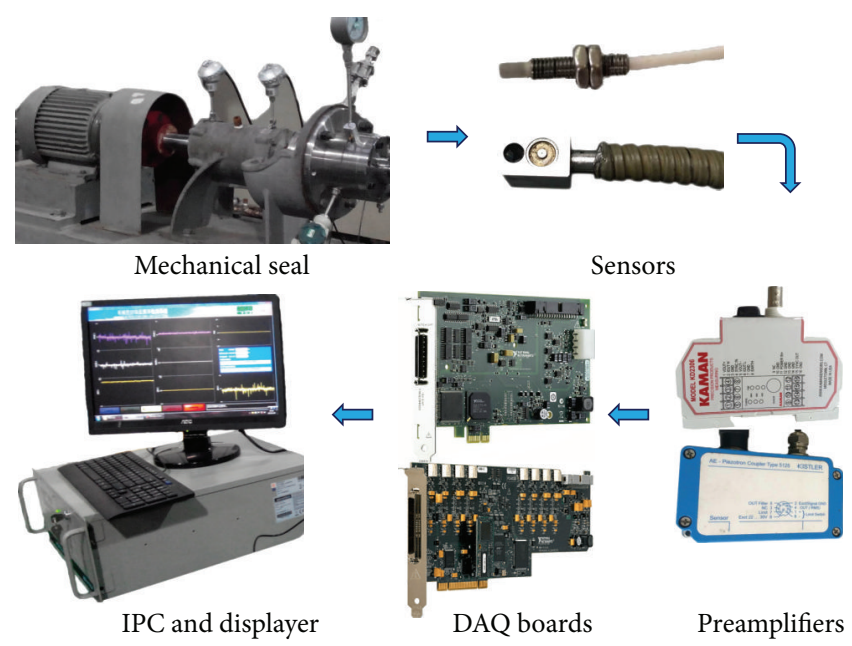

Figure 2: The monitoring system.

An AE sensor of 8152B12SP type is mounted on the shell of the seal cavity. Its effective frequency ranges from $50 \mathrm{KHz}$ to $500 \mathrm{KHz}$. To achieve high sampling rate, the data generated by $\mathrm{AE}$ sensor is acquired by a high-frequency DAQ board, and the sampling frequency is set to $1 \mathrm{MHz}$.

Finally, based on PCI data bus, the two-channel signals can be transmitted into the industrial personal computer (IPC).

The monitoring system of this experiment is given by Figure 2.

For comparing end faces signal with background noise, we operate the experiment as follows.

Step 1. Start the seal under $0.5 \mathrm{MPa}$, then raise the spindle speed up to $1500 \mathrm{rpm}$ linearly, and save the measured data into IPC.

Step 2. Repeat Step 1 several more times, so that enough data samples are collected.

Step 3. Start the seal without seal rings, then raise the spindle speed up to $1500 \mathrm{rpm}$ linearly, and save the measured data into IPC.

3.2. Signal Observation. According to the experimental data, the eddy current curve and its corresponding $\mathrm{AE}$ signal during the seal startup are given by Figure 3 .

In Figure 3, there are three contact states that can be observed.

(1) Face-contact: since the fluid film is not formed, contact friction occurs between the end faces and it makes the AE energy powerful.

(2) Face-opening: the film is not yet fully formed, so random contact occurs between the end faces and causes the AE energy sharply fluctuating.

(3) Face-separate: the film has been well formed, and fluid friction between the end faces weakens the energy and makes it stable. 


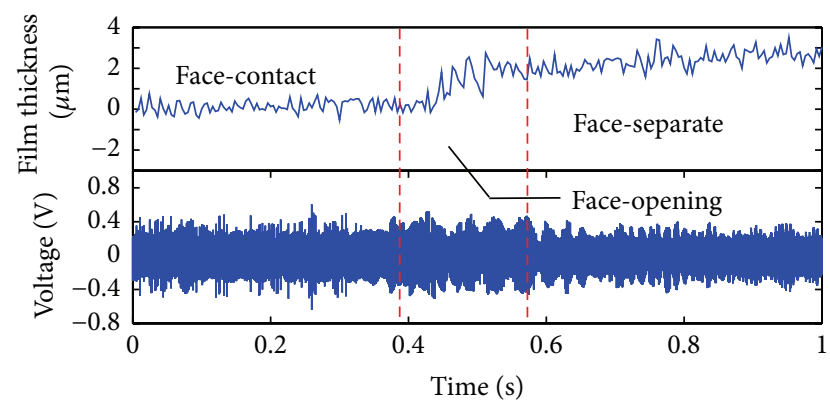

FIgURE 3: The signals during the seal startup.

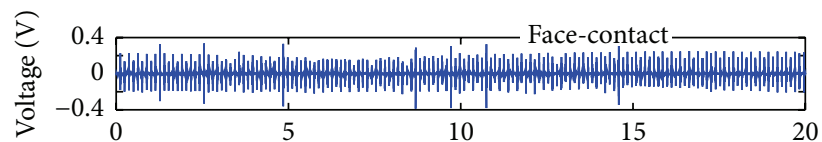

Time (ms)
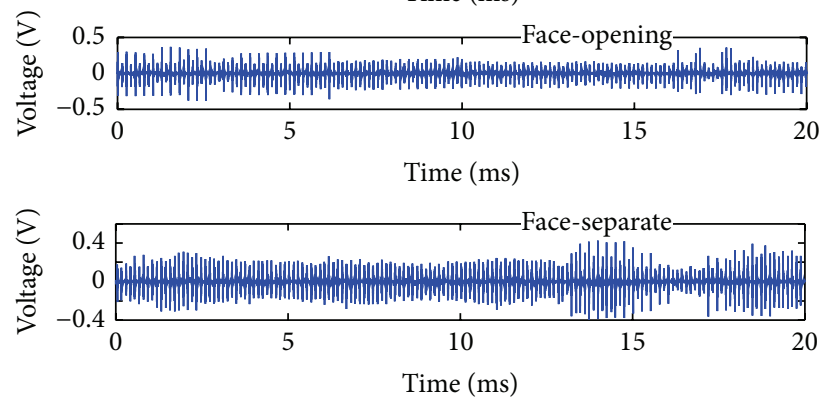

FIgURE 4: The AE signals in different contact states.

Based on the above classification, Figure 4 shows the AE signals in the different contact states.

Due to noise interference, there are some strong random fluctuations that can be observed in Figure 4. They make the signal energy unstable and bring difficulties to feature analysis and state recognition.

To further observe the effect of the noise on the signals, Figure 5 gives the AE RMS at different spindle speed.

Noise energy can be estimated by the signal collected without seal rings. Figure 5 suggests that the spindle is the main noise source, and the signal noise ratio (SNR) decreases with the increase of the spindle speed. When the seal is exceeding $1200 \mathrm{rpm}$, the observed signal is almost spindle noise.

\section{Signal Denoising}

Based on noise study, AR-GPF is presented for signal denoising. In this algorithm, AR model is used to build the DSS of the AE signal, and GPF is used for signal filtering.

4.1. Theory of AR-GPF. PF is a filtering algorithm based on Bayesian estimation and Monte Carlo method. Its core idea is to extract a series of random samples from DSS to simulate the probability density function (PDF) of system state. In this algorithm, integral is replaced by sample expectation, and the samples extracted from the DSS are called "particles" [17].

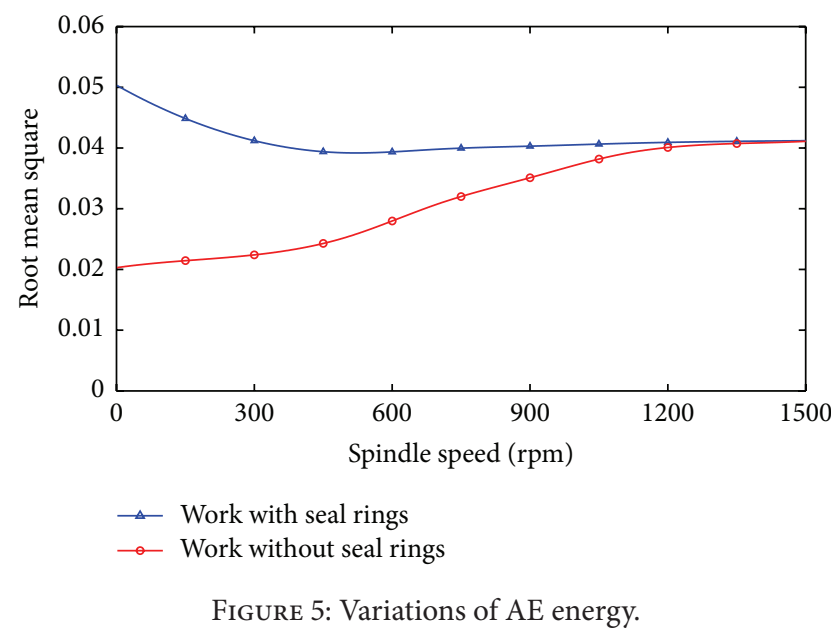

The DSS of a system can be expressed as

$$
\begin{aligned}
& x_{t}=f\left(x_{t-1}, w_{t}\right), \\
& z_{t}=h\left(x_{t}, v_{t}\right),
\end{aligned}
$$

where $f(\cdot)$ is a state function with process noise $w_{t}$ and $h(\cdot)$ is an observation function with observation noise $v_{t}$. They describe the system at time $t$ by its true state $x_{t}$ and observed value $z_{t}$.

GPF is an improved algorithm. It replaces the resampling by genetic algorithm to solve the problem of particle diversity reduction through three steps of selection, crossover, and mutation $[18,19]$. For those selected particles, the improvement based on genetic algorithm can be described as follows.

(1) Crossover. For any two particles $\left(x_{t}^{(i)}, x_{t}^{(j)}\right)$ from the particle set, whose weights are $\left(\omega_{t}^{(i)}, \omega_{t}^{(j)}\right)$, the crossover equations can be given by

$$
\begin{aligned}
& \tilde{x}_{t}^{(i)}=\alpha x_{t}^{(i)}+(1-\alpha) x_{t}^{(j)}, \\
& \tilde{x}_{t}^{(j)}=\beta x_{t}^{(j)}+(1-\beta) x_{t}^{(i)},
\end{aligned}
$$

where $\alpha, \beta$ are crossing coefficients, $\alpha=\omega_{t}^{(i)} /\left(\omega_{t}^{(i)}+\omega_{t}^{(j)}\right), \beta=$ $\omega_{t}^{(j)} /\left(\omega_{t}^{(i)}+\omega_{t}^{(j)}\right)$. If $p\left(z_{t} \mid \widetilde{x}_{t}^{(i)}\right)>\max \left\{p\left(z_{t} \mid x_{t}^{(i)}\right), p\left(z_{t} \mid x_{t}^{(j)}\right)\right\}$, $x_{t}^{(i)}$ is replaced by $\tilde{x}_{t}^{(i)}$, or $\tilde{x}_{t}^{(i)}$ is accepted with probability $p_{c}$ :

$$
p_{c}=\frac{c_{1}\left(\omega_{\max }-\omega_{c}\right)}{\left(\omega_{\max }-\omega_{\mathrm{avg}}\right)},
$$

where $c_{1}$ is a constant no greater than $1, \omega_{\max }$ is the maximum weight of the particle set, $\omega_{\text {avg }}$ is the average weight of the particle set, and $\omega_{c}$ is the larger weight of the two selected particles.

Then the same operation can be executed on $x_{t}^{(j)}$.

(2) Mutation. For any one particle $x_{t}^{(i)}$ in the particle set, its mutation equation can be given by

$$
\tilde{x}^{(i)}=x^{(i)}+\eta, \quad \eta \sim N\left(0, \omega_{\mathrm{avg}}\right) .
$$


If $p\left(z_{t} \mid \tilde{x}_{t}^{(i)}\right)>p\left(z_{t} \mid x_{t}^{(i)}\right), x_{t}^{(i)}$ is replaced by $\tilde{x}_{t}^{(i)}$, or $\tilde{x}_{t}^{(i)}$ is accepted with probability $p_{m}$ :

$$
p_{m}=\frac{c_{2}\left(\omega_{\max }-\omega_{t}^{(i)}\right)}{\omega_{\max }}
$$

where $c_{2}$ is a constant no greater than 0.5 .

To use GPF, the DSS of the system should be constructed first. We use AR model to analyze the implied time-series relation of the observed signal, so the signal sequence can be written as:

$$
Y=X A+E,
$$

where $Y=\left[x_{n+1}, x_{n+2}, \ldots, x_{m}\right]^{T}$ is the recursive sequence of the signal, $A=\left[\alpha_{1}, \alpha_{2}, \ldots, \alpha_{n}\right]^{T}$ is an autoregressive coefficient vector, $E=\left[\varepsilon_{n+1}, \varepsilon_{n+2}, \ldots, \varepsilon_{m}\right]^{T}$ is an random error vector, and $X$ is a matrix that can be given by

$$
X=\left[\begin{array}{cccc}
x_{1} & x_{2} & \cdots & x_{n} \\
x_{2} & x_{3} & \cdots & x_{n+1} \\
\vdots & \vdots & & \vdots \\
x_{m-n} & x_{m-n+1} & \cdots & x_{m-1}
\end{array}\right] .
$$

The least square estimation of $A$ can be given by

$$
A=\left(X^{T} X\right)^{-1} X^{T} Y .
$$

The optimal order $n$ of the AR model can be determined by the rule of FPE or AIC [20], so the DSS of the end faces signal can be expressed as

$$
\begin{aligned}
& x_{t}=\left(x_{t-n}, x_{t-n+1}, \ldots, x_{t-1}\right) A+w_{t}, \\
& z_{t}=x_{t}+w_{t} .
\end{aligned}
$$

Since the AE signal is observed without transformation, we assume that the observation noise is equivalent to $w_{t}$. The procedure of GPF is given as follows.

Step 1. For particle initialization, a particle set $\left\{x_{1: n}^{(i)}\right\}$ is produced according to the distribution of the measured data, in which each particle is weighted as $1 / N$. The $N$ here is the number of the sampled particles and $i$ is gotten by $i=1 \sim N$. Thus the initialized state of the signal can be estimated as

$$
\widehat{x}_{1: n}=\frac{1}{N} \sum_{i=1}^{N} x_{1: n}^{(i)}
$$

Step 2. Based on (9), the state particles $x_{t}^{(i)}$ and their observation values $z_{t}^{(i)}$ can be obtained through importance sampling. Then the corresponding weights $\omega_{t}^{(i)}$ can be calculated by

$$
\begin{aligned}
& \omega_{t}^{(i)}=\omega_{t-1}^{(i)} p\left(z_{t}^{(i)}-y_{t}\right), \\
& \omega_{t}^{(i)}=\frac{\omega_{t}^{(i)}}{\sum_{k=1}^{N} \omega_{t}^{(k)}},
\end{aligned}
$$

where $y_{t}$ is the collected data at time $t$ and $p(\cdot)$ is the PDF of the sampling space.

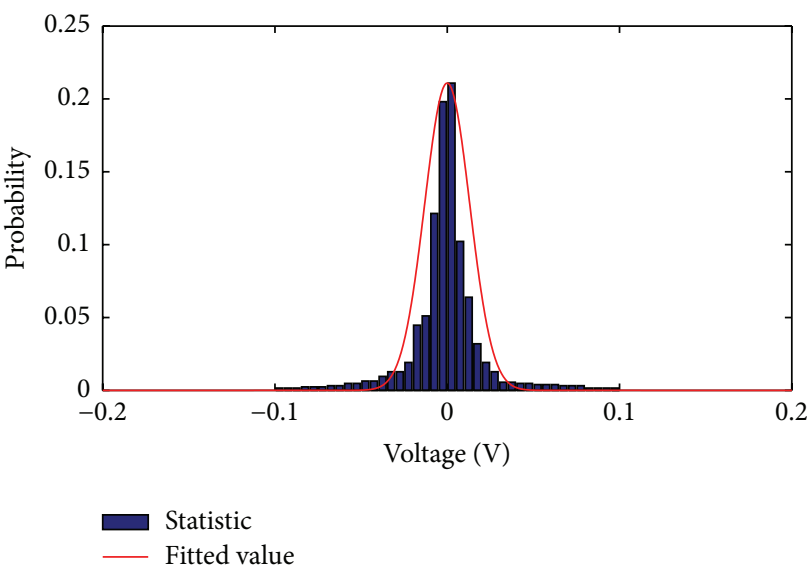

FIgURE 6: The statistical distribution of the signal noise.

Step 3. For genetic selection, $N_{\text {eff }}$ is defined as the number of effective particles, which can be gotten by

$$
N_{\text {eff }}=\frac{1}{\sum_{i=1}^{N}\left(\omega_{t}^{(i)}\right)^{2}} .
$$

If $N_{\text {eff }}$ is less than the given threshold $N_{\text {th }}$, a new set $\left\{\tilde{x}_{t}^{(i)}\right\}$ is obtained by updating those low weighted particles through genetic variation until $N_{\text {eff }} \geq N_{\text {th }}$, or $\left\{\widetilde{x}_{t}^{(i)}\right\}$ is replaced by $\left\{x_{t}^{(i)}\right\}$ directly.

Step 4. With the particles and the weights, the signal point at time $t$ can be evaluate by

$$
\widehat{x}_{t}=\sum_{i=1}^{N} \widetilde{x}_{t}^{(i)} \omega_{t}^{(i)}
$$

Then go back to Step 2 to update particles at time $t+1$.

What needs to be explained is that, by using AR analysis, the weight recursion will be much more complex than (11). But for convenience, we still assume that $\omega_{t}$ is only related to $x_{t}$ and $\omega_{t-1}$ in this paper.

4.2. AE Denoising. We extract $36 \mathrm{AE}$ data segments from every contact state, and each segment contains 20,000 data points.

For sampling particles, the noise space should be studied first. Figure 6 gives the statistic distribution of the signals collected without seal rings.

According to Figure 6, the noise obeys Gaussian distribution, and its distribution parameters at different spindle speed are estimated in Table 1.

By letting $p(\cdot)=N\left(\mu, \sigma^{2}\right)$ in (11), we sample the state particles based on the fitted normal distributions. Since the sampling function is an approximation of the real noise space, AR-GPF can effectively reduce the signal noise in time domain.

The processed signals are given by Figure 7 .

From Figure 7, the noise is effectively restrained by ARGPF, which makes the signal energy more stable than before. 
TABLE 1: Noise distribution.

\begin{tabular}{|c|c|c|c|c|c|}
\hline Spindle speed (rpm) & $(0,300)$ & $(300,600)$ & $(600,900)$ & $(900,1200)$ & $(1200,1500)$ \\
\hline & $\mu=0$ & $\mu=0$ & $\mu=0$ & $\mu=0$ & $\mu=0$ \\
\hline$N(\mu, \sigma)$ & $\sigma=0.210$ & $\sigma=0.225$ & $\sigma=0.235$ & $\sigma=0.245$ & $\sigma=0.250$ \\
\hline
\end{tabular}
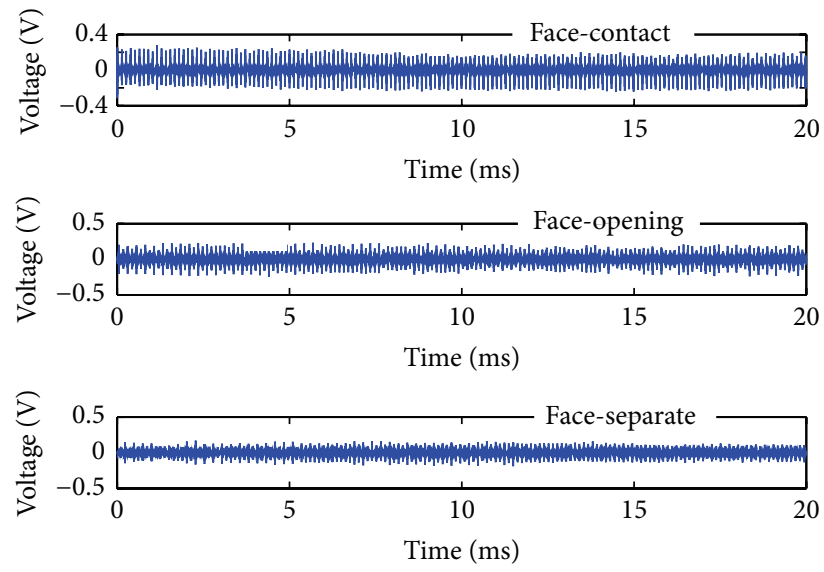

FIgURE 7: The filtered signals in different contact states.

\section{Contact State Recognition}

Seven features are extracted to describe the characteristics of the different states, and a model based on HSSVM is constructed for state monitoring.

5.1. Theory of HSSVM. SVM is a general machine learning method based on statistical learning theory. Its core idea is to construct an optimal separating hyper plane (OSH) in a higher dimensional sample space to solve the problem of nonlinear classification. Since the principle of structural risk minimization is applied, SVM usually has advantage on small-sample recognition $[21,22]$.

HSSVM is a variation of SVM. It replaces the OSH by a minimum hypersphere to wrap the samples belonging to the same class [23]. The classification sketch of HSSVM is given by Figure 8 .

For an $n$-dimensional sample space $\left\{x_{i}, y_{i}\right\}$, in which $i=$ $1 \sim m, y_{i} \in\{1,-1\}$, and $y_{i}$ is the classifying label of $x_{i}$. The classification model can be given as

$$
\begin{array}{ll}
\min _{R, a} & R^{2}+c \sum_{i=1}^{l} \xi_{i}, \quad \xi_{i} \geq 0 \\
\text { s.t. } & \left\|\phi\left(x_{i}\right)-a\right\|^{2} \leq R^{2}+\xi_{i}, \quad y_{i}=1,
\end{array}
$$

where $a$ is the sphere center, $R$ is the radius of the sphere, $l$ is the sample size, $\phi(\cdot)$ is a high-dimensional projection function, $c$ is a penalty factor, and $\xi_{i}$ is a slack variable. By using Lagrange theorem, we construct the Lagrangian as

$$
\begin{aligned}
L\left(R, a, \xi_{i}, \alpha_{i}, \beta_{i}\right)= & R^{2}+c \sum_{i=1}^{l} \xi_{i}-\sum_{i=1}^{l} \beta_{i} \xi_{i} \\
& -\sum_{i=1}^{l} \alpha_{i}\left(R^{2}+\xi_{i}-\left\|\phi\left(x_{i}\right)-a\right\|^{2}\right),
\end{aligned}
$$

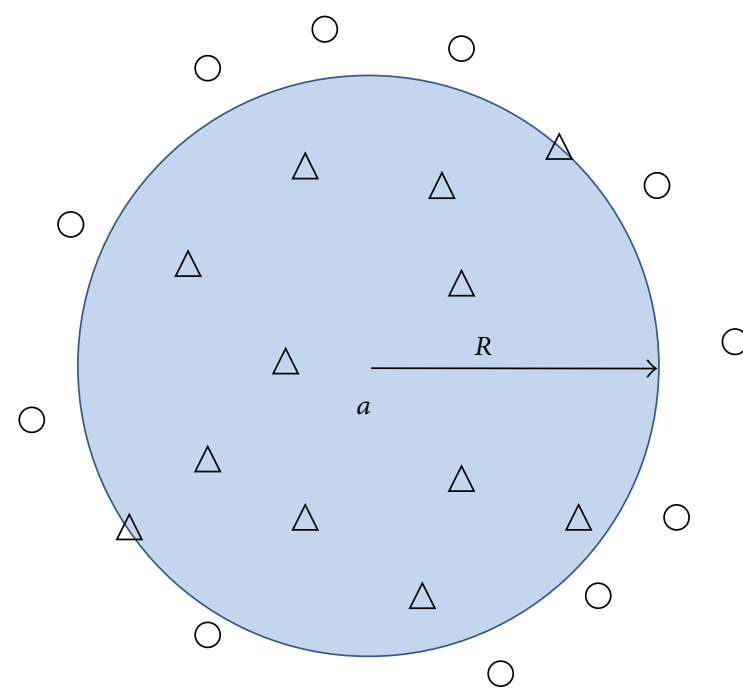

FIGURE 8: Sketch of HSSVM.

where $\alpha_{i} \geq 0$ and $\beta_{i} \geq 0$ are Lagrange multipliers. Then the optimization problem can be turned into

$$
\begin{aligned}
\min _{\alpha_{i}} & \sum_{i=1, j=1}^{l} \alpha_{i} \alpha_{j}\left\langle\phi\left(x_{i}\right) \cdot \phi\left(x_{j}\right)\right\rangle-\sum_{i=1}^{l} \alpha_{i}\left\langle\phi\left(x_{i}\right) \cdot \phi\left(x_{i}\right)\right\rangle \\
\text { s.t. } & \sum_{i=1}^{l} \alpha_{i}=1, \quad 0 \leq \alpha_{i} \leq c .
\end{aligned}
$$

So the distance of a random sample $x$ to the sphere center can be calculated by

$$
\begin{aligned}
D_{x}^{2}= & K(x, x)-2 \sum_{i=1}^{l} \alpha_{i} K\left(x, x_{i}\right) \\
& +\sum_{i=1, j=1}^{l} \alpha_{i} \alpha_{j} K\left(x_{i}, x_{j}\right),
\end{aligned}
$$

where $K(\cdot)$ is a kernel function which is used to replace the sample inner product in (17).

According to (18), when $x$ is located on the sphere, $D_{x}$ is the radius. Thus a sample can be classified according to whether the condition $D_{x}{ }^{2}-R^{2} \leq 0$ is satisfied.

5.2. State Recognition. According to the characteristics of the different states, we extract the following features from each data segment.

In Table $2, \widehat{x}$ is the filtered signal, $E(\cdot)$ is the expectation, and $\widehat{p}_{i}$ is the signal amplitude at frequency $f_{i}$. Table 3 gives the normalized features, where number $1 \sim$ number 36 belong to 
TABLE 2: Features extraction.

\begin{tabular}{|c|c|c|}
\hline Code name & Feature & Equation \\
\hline$\widehat{x}_{\mathrm{RMS}}$ & Root Mean Square (RMS) & $\hat{x}_{\mathrm{RMS}}=\sqrt{\frac{1}{n} \sum_{i=1}^{n} \hat{x}_{i}^{2}}$ \\
\hline$\widehat{x}_{\mathrm{EV}}$ & Energy Variance (EV) & $\widehat{x}_{\mathrm{EV}}=E\left(\hat{x}_{\mathrm{RMS}}^{2}\right)-E^{2}\left(\widehat{x}_{\mathrm{RMS}}\right)$ \\
\hline$\widehat{x}_{\mathrm{PAR}}$ & Peak to Average Ratio (PAR) & $\widehat{x}_{\mathrm{PAR}}=\frac{|\widehat{x}|_{\text {peak }}}{\widehat{x}_{\mathrm{RMS}}}$ \\
\hline$\widehat{x}_{\mathrm{PV}}$ & Peak Variance (PV) & $\widehat{x}_{\mathrm{PV}}=E\left(\widehat{x}_{\text {Peak }}^{2}\right)-E^{2}\left(\widehat{x}_{\text {Peak }}\right)$ \\
\hline$\widehat{x}_{\mathrm{AS}}$ & Absolute Skewness (AS) & $\widehat{x}_{\mathrm{AS}}=\frac{(1 / n) \sum_{i=1}^{n}\left(\left|\widehat{x}_{i}\right|-\widehat{x}_{\text {mean }}\right)^{3}}{\widehat{x}_{\mathrm{P}}^{3}}$ \\
\hline$\widehat{x}_{\mathrm{CF}}$ & Center Frequency (CF) & $\widehat{x}_{\mathrm{CF}}=\frac{\sum_{i=1}^{n} f_{i} \widehat{p}_{i}}{\sum_{i=1}^{n} \widehat{p}_{i}}$ \\
\hline$\hat{x}_{\mathrm{FV}}$ & Frequency Variance (FV) & $\widehat{x}_{\mathrm{FV}}=\frac{\sum_{i=1}^{n}\left(f_{i}-\widehat{x}_{\mathrm{CF}}\right)^{2} \widehat{p}_{i}}{\sum_{i=1}^{n} \widehat{p}_{i}}$ \\
\hline
\end{tabular}

TABLE 3: Normalized features.

\begin{tabular}{lccccccc}
\hline \multicolumn{2}{l}{ Sample number RMS } & EV & PAR & PV & AS & CF & FV \\
\hline Number 1 & 0.842 & 0.012 & 0.293 & 0.134 & 0.232 & 0.043 & 0.771 \\
Number 2 & 0.873 & 0.021 & 0.349 & 0.157 & 0.918 & 0.091 & 0.642 \\
$\vdots$ & & & & $\vdots$ & & & \\
Number 36 & 0.484 & 0.025 & 0.886 & 0.096 & 0.325 & 0.208 & 0.513 \\
Number 37 & 0.512 & 0.801 & 0.492 & 0.841 & 0.116 & 0.813 & 0.670 \\
$\vdots$ & & & & $\vdots$ & & & \\
Number 72 & 0.525 & 0.921 & 0.413 & 0.652 & 0.301 & 0.461 & 0.135 \\
Number 73 & 0.021 & 0.829 & 0.127 & 0.013 & 0.072 & 0.492 & 0.041 \\
$\vdots$ & & & & $\vdots$ & & & \\
Number 107 & 0.076 & 0.027 & 0.104 & 0.127 & 0.100 & 0.573 & 0.062 \\
Number 108 & 0.048 & 0.035 & 0.175 & 0.101 & 0.040 & 0.521 & 0.122 \\
\hline
\end{tabular}

face-contact, number 37 number 72 belong to face-opening, and number $73 \sim$ number 108 belong to face-separate. Taking the mean of the features from every 12 samples as statistical data, a histogram is given by Figure 9.

According to Figure 9, the features belonging to facecontact can be easily divided from those belonging to faceseparate. However, because of the dispersed distribution of the features, the state of face-opening is hard to be detected.

In this paper, we construct two sphere structures to wrap the features belonging to face-contact and face-separate and consider the rest features belonging to face-opening. 12 feature samples under each state are selected for model training, and the remaining are used for testing. By taking RBF as kernel function, the final results are given by Table 4 .

In order to further validate the paper's method, we deal the same data by some other methods for comparison. From Table 4, the detections for face-contact and face-separate have gotten satisfied results, but the accuracy rates on faceopening are quite different. Among them, the method based on Laplace wavelet got the lowest detection rates, and only limited improvements can be obtained by wavelet packet and RBF ANN. Cascade ANN has advantages on dealing nonstationary data, but the final detection rate on faceopening is still below $85 \%$. Compared with the above, the all

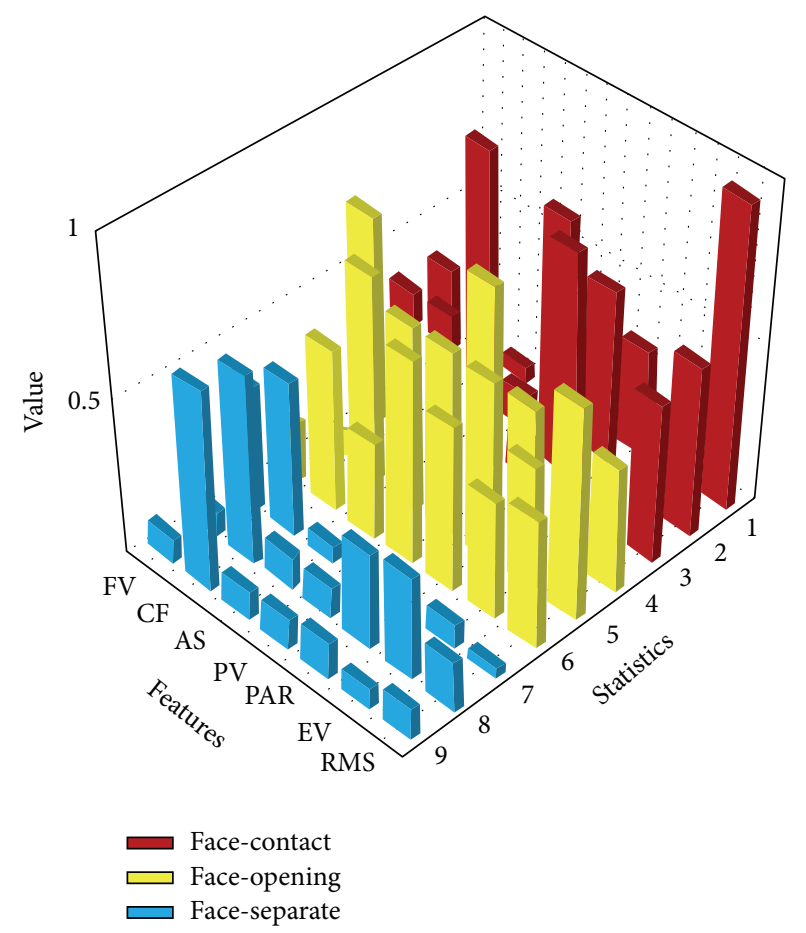

FIGURE 9: Histogram of sample features.

states are well detected by paper's method, and the average accuracy rate reaches $95 \%$.

In addition, the deficiency of this method is its lower operation speed. But at the cost of time, the method can work best in practice. In conclusion, we can get that the paper's method is very effective in contact state monitoring for seal end faces.

\section{Conclusion}

The paper studied the contact state monitoring for seal end faces by AE detection and presented a new approach based on AR-GPF and HSSVM to improve the detection rates. 
TABLE 4: The detection rates.

\begin{tabular}{|c|c|c|c|c|}
\hline \multirow{2}{*}{ Method } & \multicolumn{4}{|c|}{ Detection rate } \\
\hline & Face-contact & Face-opening & Face-separate & Average \\
\hline Paper's method & $100 \%$ & $91.7 \%$ & $95.8 \%$ & $95.8 \%$ \\
\hline Laplace wavelet with coherent coefficient & $87.5 \%$ & $29.2 \%$ & $91.7 \%$ & $69.4 \%$ \\
\hline Wavelet packet and RBF ANN & $91.7 \%$ & $54.2 \%$ & $91.7 \%$ & $79.2 \%$ \\
\hline PF and cascade ANN [24] & $95.8 \%$ & $83.3 \%$ & $95.8 \%$ & $91.7 \%$ \\
\hline
\end{tabular}

Following signal processing, some conclusions can be gotten as follows.

(1) In the AE monitoring for the seal end faces, spindle is the main noise source, and the SNR decreases with the increase of the spindle speed. When the seal exceeds $1200 \mathrm{rpm}$, the observed signal is almost noise.

(2) Based on the noise space estimation, an improved method based on AR-GPF is presented for AE denoising. For the seal end faces, AR-GPF is an excellent time-domain method for noise reduction. Data shows that it can effectively restrain the background noise of the seal and makes the signal energy more stable than before.

(3) Due to random contact, the features belonging to face-opening are distributed dispersedly. HSSVM has advantage on such features. Data shows that the final model can effectively detect the contact state of the seal end faces, and it has higher accuracy rates than some other existing methods.

\section{Conflict of Interests}

The authors declare that there is no conflict of interests regarding the publication of this paper.

\section{Acknowledgments}

This research is supported by the fund project of Nuclear Power Plant Important Pumps Mechanical Seals Achievements Transformation (National major project of science and technology achievement transformation) and the Central University Special Funding for Basic Scientific Research Business Expenses (SWJTU12CX039).

\section{References}

[1] T. Reddyhoff, R. S. Dwyer-Joyce, and P. Harper, "A new approach for the measurement of film thickness in liquid face seals," Tribology Transactions, vol. 51, no. 2, pp. 140-149, 2008.

[2] D. G. Astridge and M. D. Longfield, "Paper 11: capacitance measurements and oil film thickness in a large-radius disc and ring machine," Proceedings of the Institution of Mechanical Engineers, Conference Proceedings, vol. 182, no. 14, pp. 89-96, 1967.

[3] S. I. El-Sisi and G. S. A. Shawki, "Measurement of oil-film thickness between disks by electrical conductivity," Journal of Basic Engineering, vol. 82, no. 1, pp. 12-16, 1960.
[4] I. Etsion and I. Constantinescu, "Experimental observation of the dynamic behavior of noncontacting coned-face mechanical seals," ASLE Transactions, vol. 27, no. 3, pp. 263-270, 1984.

[5] W. B. Anderson, J. Jarzynski, and R. F. Salant, "A condition monitor for liquid lubricated mechanical seals," Tribology Transactions, vol. 44, no. 3, pp. 479-483, 2001.

[6] T. Reddyhoff, R. Dwyer-Joyce, and P. Harper, "Ultrasonic measurement of film thickness in mechanical seals," Sealing Technology, no. 7, pp. 7-11, 2006.

[7] R. Y. Li and D. He, "Rotational machine health monitoring and fault detection using EMD-based acoustic emission feature quantification," IEEE Transactions on Instrumentation and Measurement, vol. 61, no. 4, pp. 990-1001, 2012.

[8] X. Zhang, N. Z. Feng, Y. Wang, and Y. Shen, "Acoustic emission detection of rail defect based on wavelet transform and Shannon entropy," Journal of Sound and Vibration, vol. 339, pp. 419-432, 2014.

[9] L. Han, C. Li, and L. Shen, "Application in feature extraction of $\mathrm{AE}$ signal for rolling bearing in EEMD and cloud similarity measurement," Shock and Vibration, vol. 2015, Article ID 752078, 8 pages, 2015.

[10] M. Williams and N. D. Barnes, "The use of acoustic emissions for monitoring mechanical seals," in Proceedings of the 13th BPMA Pump Technical Conference, London, UK, April 1993.

[11] J. Miettinen and V. Siekkinen, "Acoustic emission in monitoring sliding contact behaviour," Wear, vol. 181-183, part 2, pp. 897900, 1995.

[12] Y. B. Fan, F. S. Gu, and A. Ball, "Acoustic emission monitoring of mechanical seals using MUSIC algorithm based on higher order statistics," Key Engineering Materials, vol. 413, pp. 811-816, 2009.

[13] E. Q. Zhang, P. Fu, Z. D. Ge, and J. K. Zhang, "Mechanical seal opening condition monitoring based on acoustic emission technology," Sensors \& Transducers Journal, vol. 172, no. 6, pp. 139-146, 2014.

[14] E. Q. Zhang, P. Fu, Z. D. Ge, Z. Zhang, and Z. P. Huang, "Study on $A E$ in mechanical seal lift-off recognition of mechanical main shaft," Sensors \& Transducers Journal, vol. 173, no. 6, pp. 158-165, 2014.

[15] Z. Gao, Y.-B. Lin, W.-F. Huang, S.-F. Suo, and Y.-M. Wang, "Acoustic emission characteristics of dry gas seals during startup," Journal of Tsinghua University, vol. 53, no. 3, pp. 319329, 2013.

[16] J. K. Zhang, E. Q. Zhang, Z. D. Zhang, and P. Fu, "Mechanical seal end faces friction condition monitoring based on particle filter," Applied Mechanics and Materials, vol. 713-715, pp. 526533, 2015.

[17] J. H. Kotecha and P. M. Djurić, "Gaussian particle filtering," IEEE Transactions on Signal Processing, vol. 51, no. 10, pp. 25922601, 2003. 
[18] M. Li, B. Pang, Y. F. He, and F. Z. Nian, "Particle filter improved by genetic algorithm and particle swarm optimization algorithm," Journal of Software, vol. 8, no. 3, pp. 666-672, 2013.

[19] W. J. Kong and Z. Wei, "Fault diagnosis of train sensors based on evolutionary genetic Particle Filter," in Proceedings of the IEEE International Conference on Intelligent Rail Transportation (ICIRT '13), pp. 255-258, IEEE, Beijing, China, September 2013.

[20] W. J. Su and H. T. Chen, "A new algorithm for long-term estimation based on AR model," Applied Mechanics and Materials, vol. 614, pp. 440-443, 2014.

[21] H.-G. Hu, X. Zhou, and Z.-M. Feng, "Fault diagnosis of rotating shaft systems based on wavelet entropy and GA-SVM," Journal of Applied Sciences, vol. 13, no. 16, pp. 3209-3214, 2013.

[22] L.-L. Jiang, H.-K. Yin, X.-J. Li, and S.-W. Tang, "Fault diagnosis of rotating machinery based on multisensor information fusion using SVM and time-domain features," Shock and Vibration, vol. 2014, Article ID 418178, 8 pages, 2014.

[23] Q. Ai and Y. P. Qin, "Feature weighted large margin and small sphere hypersphere support vector machine," ICIC Express Letters, vol. 8, no. 7, pp. 2031-2036, 2014.

[24] E. Q. Zhang, P. Fu, K. S. Li, X. H. Li, and Z. R. Zhou, "Film thickness measurement of mechanical seal based on cascaded artificial neural network recognition model," International Journal on Smart Sensing and Intelligent Systems, vol. 7, no. 4, pp. 1870-1889, 2014. 


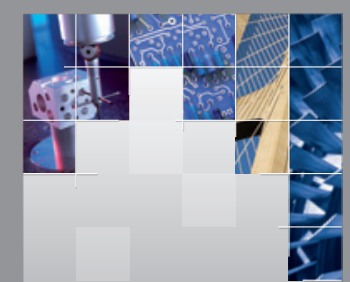

\section{Enfincering}
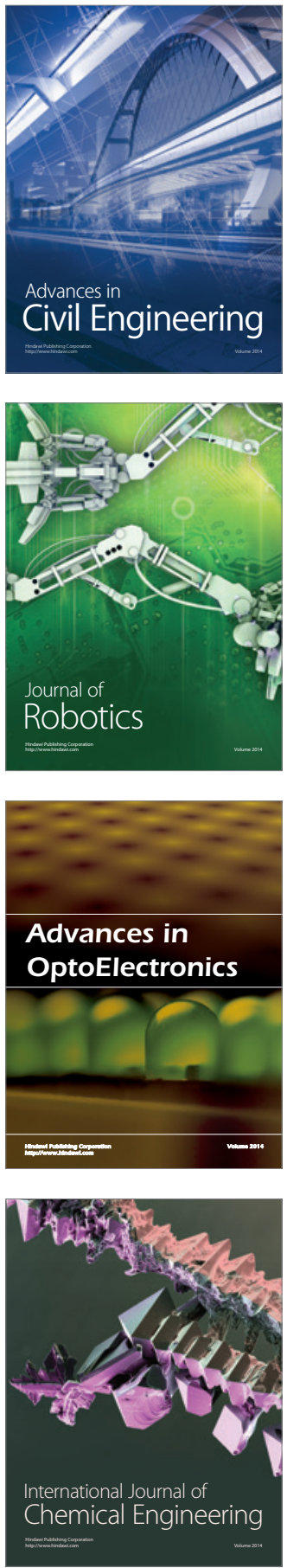

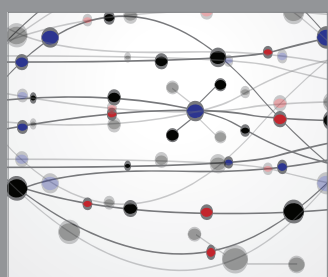

The Scientific World Journal

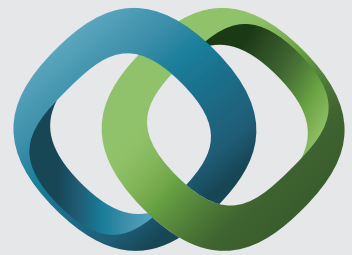

\section{Hindawi}

Submit your manuscripts at

http://www.hindawi.com
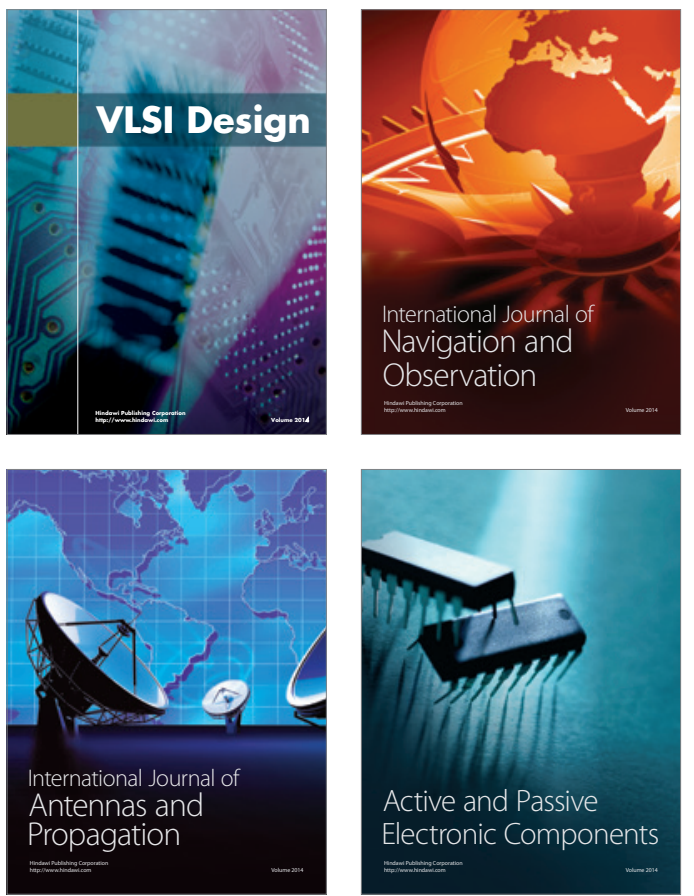
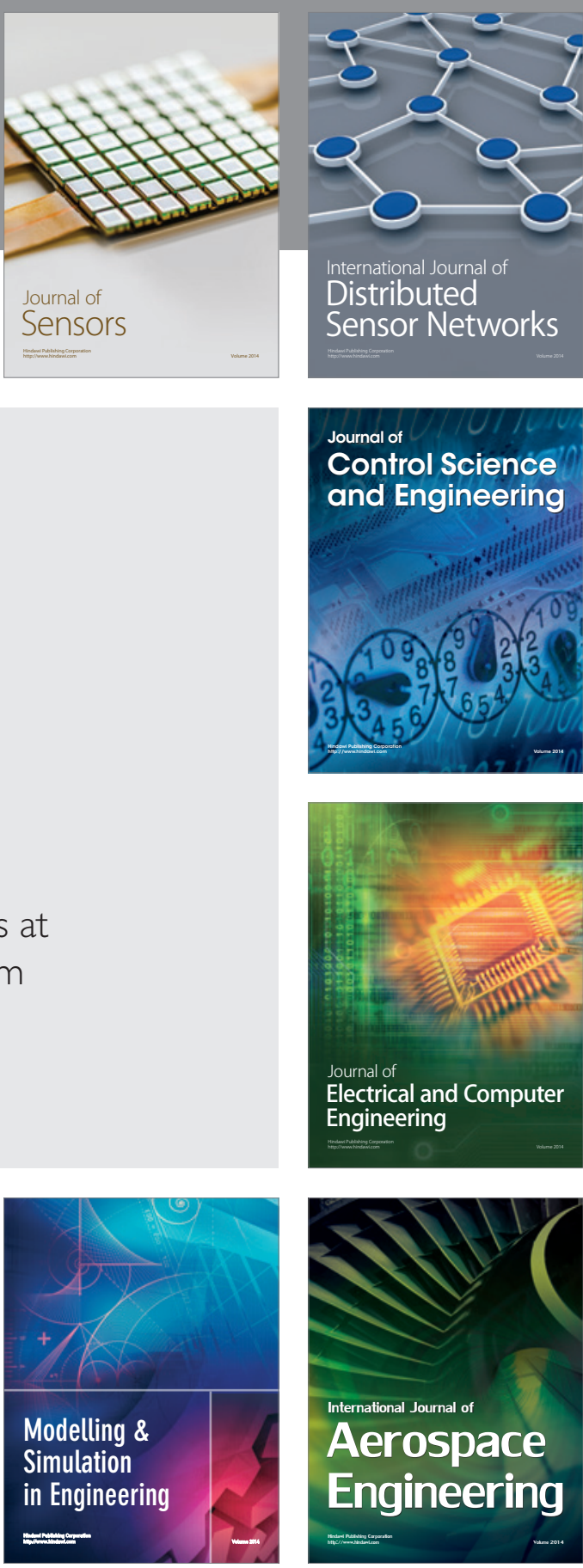

International Journal of

Distributed

Sensor Networks

Journal of

Control Science

and Engineering
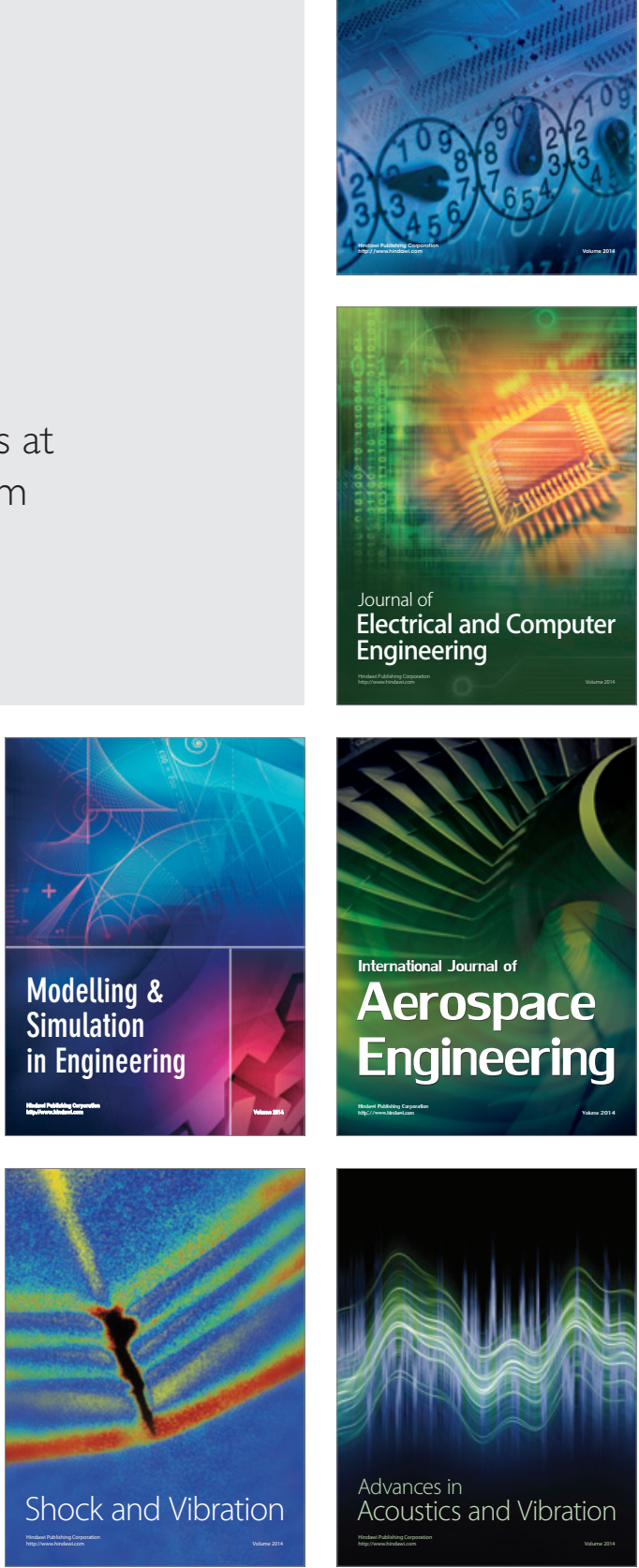